\title{
Orientação política e apoio ao isolamento social durante a pandemia da COVID-19: evidências do Brasil
}

\author{
Guilherme Ramos ${ }^{{ }^{*}}$ \\ Yan Vieites ${ }^{{ }^{*}}$ \\ Jorge Jacob ${ }^{23}$ \\ Eduardo B. Andrade 1 \\ ${ }^{1}$ Fundação Getulio Vargas / Escola Brasileira de Administração Pública e de Empresas, Rio de Janeiro / RJ - Brasil \\ ${ }^{2}$ Columbia University, New York / NY - Estados Unidos da América \\ 3 University of Virginia, Charlottesville / VA - Estados Unidos da América
}

Práticas de isolamento social têm sido amplamente recomendadas para conter a propagação da pandemia da COVID-19. No entanto, apesar do consenso médico, muitos cidadãos têm resistido a aderir e/ou apoiar a sua implementação. Enquanto essa resistência pode ter origem nos custos econômicos individuais não desprezíveis de implementar o isolamento social, argumentamos que ela também pode residir em diferenças mais fundamentais nos princípios normativos e sistemas de crenças, refletidos na orientação política. Em um estudo conduzido no Brasil, testamos a importância relativa dessas explicações ao examinar se e como o apoio ao isolamento social varia de acordo com orientação política autodeclarada e vulnerabilidade econômica pessoal. Os resultados mostram que enquanto a vulnerabilidade econômica não influencia o apoio ao isolamento social, indivíduos que se consideram de direita apoiam sistematicamente menos tais práticas do que aqueles que se consideram de esquerda. Diferenças em suas sensibilidades a ameaças ao sistema econômico ajudam a explicar o fenômeno.

Palavras-chave: COVID-19; orientação política; isolamento social, política pública.

\section{Orientación política y apoyo al aislamiento social durante la pandemia de COVID-19: evidencia de Brasil}

Las prácticas de aislamiento social se han recomendado ampliamente para contener la propagación de la pandemia de COVID-19. Sin embargo, a pesar del consenso médico, muchos ciudadanos se han resistido a adherirse y/o apoyar su implementación. Si bien esta resistencia puede provenir de costos económicos personales no despreciables en la implementación del aislamiento social, argumentamos que también puede residir en diferencias más fundamentadas en principios normativos y sistemas de creencias, reflejados en la orientación política. El artículo prueba, mediante una encuesta realizada en Brasil, la importancia relativa de estas explicaciones al examinar si y de qué manera el apoyo al aislamiento social varía de acuerdo con la orientación política declarada y la vulnerabilidad económica personal. Los resultados muestran que, si bien la vulnerabilidad económica no influye en el apoyo al aislamiento social, los conservadores apoyan dichas prácticas sistemáticamente menos que los liberales. Las diferencias de sensibilidad ante las amenazas al sistema económico ayudan a explicar el fenómeno.

Palabras clave: COVID-19; orientación política; aislamiento social; política pública.

\section{Political orientation and support for social distancing during the COVID-19 pandemic: evidence from Brazil}

Social distancing practices have been widely recommended to curb the COVID-19 pandemic. However, despite the medical consensus, many citizens have resisted adhering to and/or supporting its implementation. While this resistance may stem from the non-negligible personal economic costs of implementing social distancing, we argue that it may also reside in more fundamental differences in normative principles and belief systems, as reflected by political orientation. In a study conducted in Brazil, we test the relative importance of these explanations by examining whether and how support for social distancing varies according to self-identified political orientation and personal economic vulnerability. Results show that while economic vulnerability does not influence support for social distancing, conservatives are systematically less supportive of these practices than liberals. Discrepancies in sensitivity to threats to the economic system help explain the phenomenon.

Keywords: COVID-19; political orientation; social distancing; public policy. 


\section{INTRODUÇÃO}

No dia 10 de junho de 2020, o mundo contava 7 milhões de casos confirmados da COVID-19 e 400 mil mortes devido à doença. Em resposta à pandemia, autoridades de saúde nacionais e internacionais convergiram para recomendar a implementação, o quanto antes, de duras políticas de isolamento social para conter a propagação do coronavírus causador da doença (Tabari et al., 2020; World Health Organization, 2020). No entanto, apesar do consenso médico, muitos cidadãos têm relutado em adotar tais medidas de controle (Tanne, 2020). O presente estudo investiga o papel da orientação política dos indivíduos na definição de atitudes e comportamentos em relação às políticas de isolamento social.

A polarização política está bem viva no Brasil (Samuels \& Zucco, 2014), tendo aumentado nas últimas décadas em todo o mundo (Carothers \& O’Donohue, 2019) e demonstrando tendência de continuidade (Abramowitz \& Saunders, 2008; Mansbridge, 2016). Nos EUA, por exemplo, a parcela de pessoas identificadas como "liberal" (liberais, considerados naquele país como 'esquerda') ou "conservative" (conservadoras, ou 'direita') aumentou de 57\% em 1992 para 65\% em 2010 (Saad, 2012). À medida que a orientação política aumenta em importância, as preferências tornam-se mais claramente divididas em linhas ideológicas. Já está demonstrado, no contexto dos EUA, que liberals e conservatives têm diferentes opiniões sobre uma ampla variedade de questões, desde o controle de armas até as mudanças climáticas (Gramlich \& Schaeffer, 2019; VanBoven et al., 2018). Dado que a orientação política molda amplamente as opiniões sobre políticas públicas (Bail et al., 2018; Dimock \& Carroll, 2014), é provável que as opiniões das pessoas sobre isolamento social também podem ser influenciadas por sua posição no espectro político.

No centro das discussões sobre a adoção de medidas de isolamento social está o aparente trade-off entre saúde pública e bem-estar econômico. Em muitas regiões do mundo têm-se aceitado a premissa de que o impacto econômico brutal e duradouro de medidas de isolamento social rigorosas e antecipadas para combater a pandemia pode ser tão ou mais prejudicial à sociedade, do que optar por políticas de isolamento mais flexíveis, ou mesmo não tomar nenhuma providência nesse sentido (Snooks, 2020). Embora essa ideia tenha sido posta de lado em muitos lugares, ela ainda resiste em algumas regiões (The Lancet, 2020). Mesmo que essa resistência tenha um forte fundamento nos custos pessoais da implementação do isolamento social, ela também pode encontrar raízes em diferenças relacionadas a princípios normativos e sistemas de crença. Para testar a importância relativa dessas explicações, investigamos a associação entre o apoio dado ao isolamento social e dois indicadores: (a) a vulnerabilidade econômica (ou seja, a classe social e o impacto previsto nas finanças pessoais); e (b) orientação política, que está no cerne do suposto trade-offentre saúde pública e bem-estar econômico.

Embora as consequências, econômicas ou de saúde, do isolamento social, afetem pessoas que se reconhecem tanto à esquerda quanto à direita no espectro político, essas últimas podem ser muito mais sensíveis aos possíveis efeitos econômicos da adoção de medidas mais rígidas de isolamento social. Ao contrário daquelas à esquerda, as pessoas que se reconhecem 'de direita' consideram que empresas e indústrias são elementos-chave da sociedade (Choma et al., 2014; Kerlinger, 1984). Tendem também a endossar os valores protestantes do trabalho, que enfatizam a importância do trabalho duro e esforço contínuo (Atieh et al., 1987), e se opõem ao aumento dos gastos públicos (Jacoby, 2000; Rudolph \& Evans, 2005), o que é uma medida tradicional usada para aliviar as perdas econômicas infligidas pelo isolamento social. A hipótese levantada aqui, portanto, é a de que as pessoas que se reconhecem à direita no espectro político sejam sistematicamente menos favoráveis às práticas e 
políticas de isolamento social do que indivíduos considerados 'de esquerda'. Além disso, a associação entre orientação política e atitude em relação a políticas de isolamento social deve ser, pelo menos em parte, explicada pelas diferenças na sensibilidade dos cidadãos às ameaças ao sistema econômico.

A presente pesquisa oferece uma série de contribuições para a literatura. Em primeiro lugar, enquanto pesquisas anteriores lançaram dúvida sobre a influência da orientação política e da afiliação partidária, (Converse, 1964; Fiorina, 2006), trabalhos mais recentes reforçam o elevado poder preditivo da orientação política sobre as preferências políticas (Dimock \& Carroll, 2014; Bail et al., 2018). Considerando a gravidade do atual contexto e o relativo consenso sobre como lidar com a emergência sanitária, a pandemia da COVID-19 representa um teste crítico para a relevância da orientação política dos indivíduos na definição de seu apoio a determinadas políticas públicas. Em segundo lugar, até o momento, as pesquisas têm enfatizado o papel da percepção individual em relação aos riscos à saúde para explicar a relação entre orientação política e apoio a medidas de isolamento social (Alcott et al., 2020; Barrios \& Hochberg, 2020; Conway III et al., 2020; Painter \& Qiu, 2020; Rosenfeld et al., 2020). Nosso trabalho avança em relação a essas descobertas ao introduzir um novo mecanismo na discussão: a sensibilidade às ameaças ao sistema econômico. Por fim, embora existam evidências do efeito da orientação política na adesão a medidas de isolamento social, tais evidências têm origem predominantemente em estudos realizados com dados dos EUA. No entanto, características idiossincráticas regionais são determinantes para que uma questão se torne ou não politicamente polarizada (McCright, Dunlap, \& Marquart-Pyatt, 2016). Assim, esse estudo também contribui com a literatura ao investigar a robustez do fenômeno no Brasil.

\section{ESTUDO}

\subsection{Metodologia}

Seguindo uma tendência recente nas ciências comportamentais (Nosek et al., 2018), realizamos um estudo experimental pré-registrado. Antes de analisar os dados, submetemos nossas hipóteses e o plano analítico a um registro independente (https://aspredicted.org/blind.php? $\mathrm{x}=4 \mathrm{er} 5 \mathrm{ui}$ ). Embora não seja uma panaceia (Yamada, 2018), o pré-registro representa uma prática importante na academia, na medida em que confere credibilidade à pesquisa, reduzindo práticas questionáveis como a elaboração de hipóteses após os resultados serem conhecidos e o p-hacking (Miguel et al., 2014; Munafò et al., 2017; Nosek et al., 2018; Yamada, 2018).

Conforme descrito no relatório do pré-registro, testamos a importância relativa da orientação política e da vulnerabilidade econômica (por exemplo, classe social, impacto previsto nos ganhos pessoais) na predição do apoio dos indivíduos ao isolamento social. Embora não antecipado no pré-registro, também conduzimos análises de mediação exploratórias para avaliar se o efeito estudado pode ser explicado pela sensibilidade dos indivíduos a ameaças ao sistema econômico. Além disso, no protocolo, também inserimos hipóteses sobre três intervenções diferentes desenhadas para reforçar o apoio ao isolamento social. No entanto, como nenhuma dessas intervenções influenciou sistematicamente atitudes e comportamentos, seguimos as sugestões da equipe de revisão para colocar menos ênfase nessas intervenções ao elaborar o presente artigo, apresentando-as no apêndice. 
Participantes. Os participantes foram recrutados no Brasil através das redes de relacionamento e mídias sociais dos autores entre os dias 24 e 27 de março de 2020. Como recompensa pela participação, anunciamos que doaríamos $\mathrm{R} \$ 1,00$ por participante a uma causa social relacionada à mitigação dos impactos da COVID-19. Embora 1.053 pessoas tenham participado do estudo, apenas 768 delas atenderam a todos os critérios de inclusão na amostra estabelecidos no pré-registro. A exclusão do participante se deu quando ele não concluiu todas as etapas do estudo ou escolheu a opção "não sei" em qualquer uma das variáveis dependentes.

Procedimento. Após o consentimento, os participantes foram aleatoriamente designados para uma das quatro condições experimentais, que faziam parte da hipótese elaborada no apêndice. Em seguida, todos procederam aos itens referentes às variáveis dependentes. Primeiro, perguntamos o quanto eles apoiavam a adoção de práticas de isolamento social por cerca de um mês (sendo 1 para 'fortemente contra', e 4 para 'fortemente a favor'). Depois, perguntamos quão rigorosas as políticas governamentais de isolamento social deveriam ser (sendo 1 para 'nenhuma política', e 4 para 'política rigorosa e imediata'). Em terceiro lugar, os participantes foram solicitados a expressar uma medida consequencial, indicando qual tipo de causa eles gostariam que recebesse, em forma de doação, o valor de contrapartida de sua participação (sendo 1 para uma causa destinada a mitigar os impactos da pandemia na saúde, e 0 para uma causa relacionada a mitigar os impactos da COVID-19 na economia). Enquanto as duas primeiras variáveis dependentes estavam relacionadas a questões atitudinais, a terceira capturou um comportamento consequencial que refletia mais explicitamente o trade-off entre economia e saúde pública.

Em seguida, os participantes preencheram um questionário sociodemográfico, onde foi incorporada uma escala de 4 pontos de orientação política. Adotamos 'de esquerda' e 'de direita' nos questionários, como correspondentes, respectivamente, aos termos "liberal" e "conservative" usados na literatura internacional (Hasson et al., 2018; Jost, Federico, \& Napier, 2009). A questão para essa caracterização dos respondentes dizia: "Em termos de orientação política, como você se define?" (sendo 1 para 'claramente de esquerda', 4 para 'claramente de direita', e incluindo a opção 'não sei o que significa ser de esquerda ou de direita'). Além disso, esse questionário sociodemográfico também incluiu: (a) uma medida do impacto previsto na renda pessoal, caso o participante tenha que fazer isolamento social (sendo 1 para 'afetado pelo menos parcialmente', 0 para 'não afetado', e incluindo a opção 'não tenho condições de prever'); (b) uma medida subjetiva para aferição de classe social, com 5 pontos (sendo 1 'minha renda está muito abaixo da média da população brasileira' e 5 sendo 'muito acima da média'); e (c) perguntas sobre educação e renda que, baseadas na literatura (Adler et al., 2000; Korndörfer, Egloff, \& Schmukle, 2015), foram padronizadas e consolidadas em uma única medida de classe social objetiva. Enquanto a orientação política reflete sistemas de crenças e princípios normativos das pessoas, a previsão individual sobre o impacto nos ganhos e a classe social dos respondentes representaram medidas de vulnerabilidade econômica.

Por fim, perguntamos aos participantes sobre a dimensão de suas vidas pessoais que provavelmente seria a mais afetada pela pandemia (sendo 1 para 'finanças', 0 para 'saúde ou segurança'). Perguntamos o mesmo sobre a vida de outras pessoas próximas (por exemplo, família, vizinhos, comunidade) e da sociedade em geral. Essas variáveis procuraram capturar, respectivamente, a sensibilidade relativa a ameaças às finanças pessoais, às finanças de pessoas próximas, e às finanças do sistema econômico 
como um todo. Além disso, coletamos medidas de exposição ao coronavírus, bem como outras questões demográficas usuais (e.g., idade, sexo, etnia, estado civil, região geográfica) apenas para fins descritivos. Com isso, agradecemos a atenção dos participantes e encerramos o questionário.

Plano de análise. Testamos os efeitos da orientação política, classe social e impacto esperado nos ganhos pessoais nas três variáveis dependentes: decisão de doar para causas relacionadas à saúde (versus doar para causas relacionadas a economia) e apoio às práticas e políticas de isolamento social. Utilizamos regressões logísticas para a variável 'decisão de doar' e modelos de regressão linear para as duas variáveis referentes ao isolamento social. Para investigar o papel mediador da sensibilidade relativa a ameaças ao sistema econômico e compará-lo com o efeito de ameaças a si próprio e à comunidade, realizamos regressões aparentemente não relacionadas, que permitem uma estimativa simultânea de equações.

\section{RESULTADOS E DISCUSSÃ0}

Descrição de dados. A Tabela 1 exibe as estatísticas descritivas. Nossa amostra é composta por uma maioria de mulheres, pessoas brancas e de classe alta, residentes nas regiões sul e sudeste do Brasil. Quando o estudo foi realizado, apenas uma pequena porcentagem dos participantes havia sido testada para o COVID-19, mas 16\% deles conheciam alguém que havia testado positivo. Além disso, os participantes pareciam estar bem distribuídos ao longo do espectro político. Um teste de Shapiro-Wilk sobre nossa medida de orientação política sugere que os participantes seguiram uma distribuição normal $(W=1,00 ; \mathrm{z}=-1,49 ; p=0,93)$.

\section{TABELA 1 ESTATÍSTICAS DESCRITIVAS}

\begin{tabular}{|c|c|c|c|c|c|}
\hline Variáveis & Média & DV & Min & Max & $\mathrm{N}^{0}$ \\
\hline \multicolumn{6}{|l|}{ Controle } \\
\hline Homem & 0,39 & 0,49 & 0 & 1 & 768 \\
\hline Idade & 37,81 & 12,46 & 18 & 72 & 768 \\
\hline Branco & 0,79 & 0,41 & 0 & 1 & 768 \\
\hline Religião & 2,86 & 1,48 & 1 & 5 & 768 \\
\hline Casado & 0,48 & 0,50 & 0 & 1 & 768 \\
\hline Região Sudeste & 0,67 & 0,47 & 0 & 1 & 768 \\
\hline Região Sul & 0,20 & 0,40 & 0 & 1 & 768 \\
\hline Região Nordeste & 0,07 & 0,26 & 0 & 1 & 768 \\
\hline Trabalhador da saúde & 0,21 & 0,41 & 0 & 1 & 768 \\
\hline Testado para coronavírus & 0,03 & 0,18 & 0 & 1 & 768 \\
\hline Resultado positivo & 0,00 & 0,04 & 0 & 1 & 768 \\
\hline Conhecidos que apresentaram resultado positivo & 0,16 & 0,37 & 0 & 1 & 768 \\
\hline
\end{tabular}




\begin{tabular}{l|c|c|c|c|c} 
Variáveis & Média & DV & Min & Max & No $^{\circ}$ \\
Variáveis Independentes & & & & & \\
$\quad$ Orientação política (escala) & 2,46 & 0,95 & 1 & 4 & 747 \\
$\quad$ Previsão de impacto na renda & 0,39 & 0,49 & 0 & 1 & 543 \\
Classe social subjetiva & 4,13 & 1,04 & 1 & 5 & 774 \\
Classe social objetiva & 0,00 & 0,80 & $-3,70$ & 3,84 & 730 \\
Educação em no anos & 16,66 & 1,82 & 5 & 18 & 774 \\
Renda domiciliar & 14789 & 11322 & 500 & 40000 & 730 \\
\hline Variáveis Dependentes & & & & & \\
$\quad$ Apoio às práticas de isolamento social & 3,49 & 0,74 & 1 & 4 & 768 \\
Apoio às políticas de isolamento social & 3,58 & 0,64 & 1 & 4 & 768 \\
Doar para saúde (versus economia) & 0,70 & 0,46 & 0 & 1 & 768 \\
\hline
\end{tabular}

Fonte: Elaborada pelos autores.

Análises dos dados. Como mencionado na seção anterior, usamos a regressão logística para a análise da variável de doação e a regressão linear para as duas variáveis relacionadas ao isolamento social. Assim, cada uma dessas variáveis foi submetida a uma regressão, em que incluímos orientação política, classe social objetiva e subjetiva, e previsão de impacto na renda pessoal como variáveis independentes. Para evitar o viés de variável omitida e oferecer estimativas mais precisas, controlamos por idade, sexo, raça, religião e estado civil dos participantes, bem como sua região geográfica de residência, se trabalhou no setor de saúde, se amigos ou familiares testaram positivo para COVID-19 e dia de participação no estudo. Diferentes especificações foram apresentadas para atestar a robustez dos resultados.

Surpreendentemente, nem a classe social nem a previsão de impacto na renda pessoal influenciaram de forma consistente o apoio ao isolamento social e/ou às decisões sobre a causa para qual doar, se saúde ou economia (veja a Tabela 2). Por outro lado, e conforme esperado, a orientação política foi capaz de prever, sistematicamente, $\mathrm{o}$ apoio ao isolamento social e às decisões sobre doar. Como mostra a figura 1, quanto mais os participantes se identificaram como 'de direita', menos apoiaram a prática $(\beta=-0,30 ; t(498)=-8,91 ; p<0,001)$ e as políticas $(\beta=-0,23 ; t(498)=-7,43 ; p<0,001)$ de isolamento social. Esses respondentes também foram menos propensos a doar para a causa relacionada à saúde $(\beta=-0,21 ; z=-1,85 ; p=0,06)$. Os resultados demonstraram que, entre os preditores, a orientação política teve o efeito mais forte para antever o apoio dos indivíduos às práticas e políticas de isolamento social ${ }^{1}$, inclusive um efeito mais pronunciado do que o fator de vulnerabilidade econômica.

\footnotetext{
${ }^{1}$ Rodamos novamente os modelos 6 e 9 usando coeficientes padronizados para analisar a importância relativa de cada varável para explicar o apoio ao isolamento social. A orientação política foi de longe o preditor mais forte $\left(\beta_{\text {práticas }}=-0,37 ; \beta_{\text {políticas }}=-0,34\right)$, com o segundo colocado alcançando cerca da metade de seu efeito.
} 


\section{TABELA 2 EFEITOS DA ORIENTAÇÃO POLÍTICA NO APOIO AO ISOLAMENTO SOCIAL E DOAÇÃO PARA CAUSAS RELACIONADAS A SAÚDE}

\begin{tabular}{|c|c|c|c|c|c|c|c|c|c|}
\hline & \multicolumn{9}{|c|}{ Variáveis dependentes } \\
\hline & \multicolumn{3}{|c|}{$\begin{array}{l}\text { Doar para saúde (versus } \\
\text { economia) }\end{array}$} & \multicolumn{3}{|c|}{$\begin{array}{l}\text { Apoio às práticas de isolamento } \\
\text { social }\end{array}$} & \multicolumn{3}{|c|}{$\begin{array}{c}\text { Apoio às políticas de isolamento } \\
\text { social }\end{array}$} \\
\hline & \multicolumn{3}{|c|}{ Regressão Logística } & \multicolumn{3}{|c|}{ MMQ } & \multicolumn{3}{|c|}{ MMQ } \\
\hline & (1) & $(2)$ & (3) & (4) & (5) & (6) & (7) & (8) & (9) \\
\hline $\begin{array}{l}\text { Orientação política } \\
\text { (escala) }\end{array}$ & $\begin{array}{c}-0,34^{\star \star \star} \\
(0,09)\end{array}$ & $\begin{array}{c}-0,26^{\star \star} \\
(0,10)\end{array}$ & $\begin{array}{l}-0,21^{*} \\
(0,12)\end{array}$ & $\begin{array}{c}-0,35^{\star \star \star} \\
(0,03)\end{array}$ & $\begin{array}{c}-0,38^{\star \star \star} \\
(0,03)\end{array}$ & $\begin{array}{c}-0,30^{\star \star \star} \\
(0,03)\end{array}$ & $\begin{array}{c}-0,23^{\star \star \star} \\
(0,02)\end{array}$ & $\begin{array}{c}-0,26^{\star \star \star} \\
(0,03)\end{array}$ & $\begin{array}{c}-0,23^{\star \star \star} \\
(0,03)\end{array}$ \\
\hline Classe social subjetiva & & $\begin{array}{l}-0,14 \\
(0,13)\end{array}$ & $\begin{array}{l}-0,08 \\
(0,14)\end{array}$ & & $\begin{array}{l}0,01 \\
(0,04)\end{array}$ & $\begin{array}{l}0,00 \\
(0,04)\end{array}$ & & $\begin{array}{l}0,06^{\star} \\
(0,03)\end{array}$ & $\begin{array}{l}0,05 \\
(0,04)\end{array}$ \\
\hline Classe social objetiva & & $\begin{array}{l}-0,01 \\
(0,14)\end{array}$ & $\begin{array}{l}0,00 \\
(0,15)\end{array}$ & & $\begin{array}{l}-0,05 \\
(0,04)\end{array}$ & $\begin{array}{l}-0,03 \\
(0,04)\end{array}$ & & $\begin{array}{l}0,00 \\
(0,04)\end{array}$ & $\begin{array}{l}0,00 \\
(0,04)\end{array}$ \\
\hline $\begin{array}{l}\text { Previsão de impacto na } \\
\text { renda }\end{array}$ & & $\begin{array}{l}-0,17 \\
(0,20)\end{array}$ & $\begin{array}{l}-0,17 \\
(0,21)\end{array}$ & & $\begin{array}{c}-0,13^{\star \star} \\
(0,06)\end{array}$ & $\begin{array}{l}-0,10 \\
(0,06)\end{array}$ & & $\begin{array}{l}0,02 \\
(0,06)\end{array}$ & $\begin{array}{l}0,00 \\
(0,06)\end{array}$ \\
\hline Idade & & & $\begin{array}{l}0,01 \\
(0,01)\end{array}$ & & & $\begin{array}{l}-0,00 \\
(0,00)\end{array}$ & & & $\begin{array}{l}-0,00 \\
(0,00)\end{array}$ \\
\hline Homem & & & $\begin{array}{l}-0,06 \\
(0,21)\end{array}$ & & & $\begin{array}{c}-0,29^{\star \star \star} \\
(0,06)\end{array}$ & & & $\begin{array}{c}-0,16^{\star \star \star} \\
(0,06)\end{array}$ \\
\hline Branco & & & $\begin{array}{l}-0,26 \\
(0,27)\end{array}$ & & & $\begin{array}{l}0,01 \\
(0,08)\end{array}$ & & & $\begin{array}{l}0,18^{* *} \\
(0,07)\end{array}$ \\
\hline Religião & & & $\begin{array}{l}0,03 \\
(0,07)\end{array}$ & & & $\begin{array}{l}-0,01 \\
(0,02)\end{array}$ & & & $\begin{array}{l}0,02 \\
(0,02)\end{array}$ \\
\hline Casado & & & $\begin{array}{c}-0,54^{\star \star} \\
(0,22)\end{array}$ & & & $\begin{array}{l}-0,05 \\
(0,06)\end{array}$ & & & $\begin{array}{l}-0,10 \\
(0,06)\end{array}$ \\
\hline Trabalhador da saúde & & & $\begin{array}{l}0,24 \\
(0,25)\end{array}$ & & & $\begin{array}{l}-0,07 \\
(0,07)\end{array}$ & & & $\begin{array}{l}0,04 \\
(0,07)\end{array}$ \\
\hline $\begin{array}{l}\text { Conhecidos que } \\
\text { apresentaram resultado } \\
\text { positivo }\end{array}$ & & & $\begin{array}{l}0,11 \\
(0,27)\end{array}$ & & & $\begin{array}{l}-0,03 \\
(0,08)\end{array}$ & & & $\begin{array}{l}-0,05 \\
(0,07)\end{array}$ \\
\hline Efeitos da região & $\operatorname{Sim}$ & $\operatorname{Sim}$ & $\operatorname{Sim}$ & Sim & $\operatorname{Sim}$ & $\operatorname{Sim}$ & $\operatorname{Sim}$ & Sim & $\operatorname{Sim}$ \\
\hline Efeitos do tempo & $\operatorname{Sim}$ & Sim & Sim & Sim & $\operatorname{Sim}$ & $\operatorname{Sim}$ & $\operatorname{Sim}$ & Sim & $\operatorname{Sim}$ \\
\hline$N^{0}$ de observações & 747 & 514 & 514 & 747 & 514 & 514 & 747 & 514 & 514 \\
\hline R-quadrado & & & & 0,20 & 0,25 & 0,32 & 0,12 & 0,15 & 0,20 \\
\hline Log-probabilidade & $-452,32$ & $-313,77$ & $-308,29$ & & & & & & \\
\hline Crit. de Info. de Akaike & 908,64 & 637,53 & 648,57 & & & & & & \\
\hline
\end{tabular}

Nota: Erro padrão entre parênteses. ${ }^{* *} \mathrm{p}<0,1 ;{ }^{* *} \mathrm{p}<0,05 ;{ }^{*} \mathrm{p}<0,10$.

Fonte: Elaborada pelos autores. 

NA ATITUDE DE DOAR PARA CAUSAS RELACIONADAS À SAÚDE

Painel A:

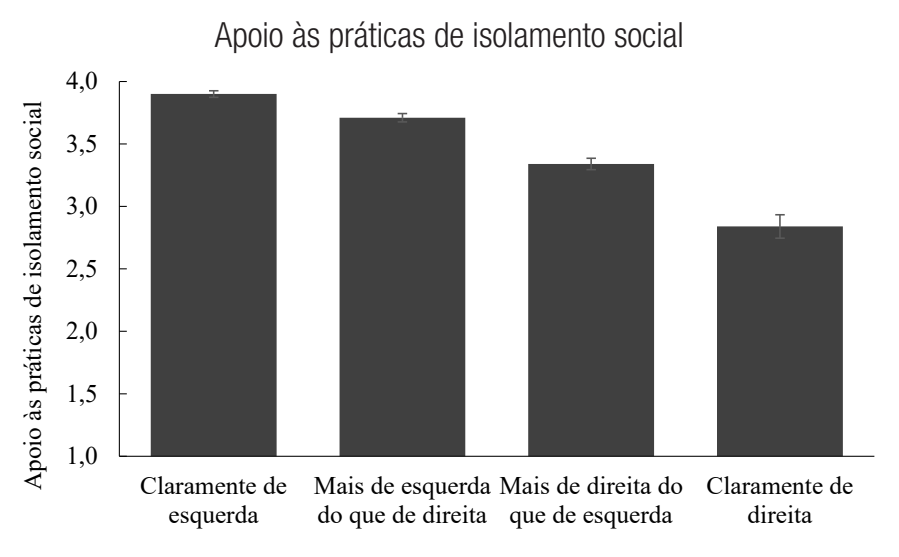

Painel B:

Apoio às políticas públicas de isolamento social

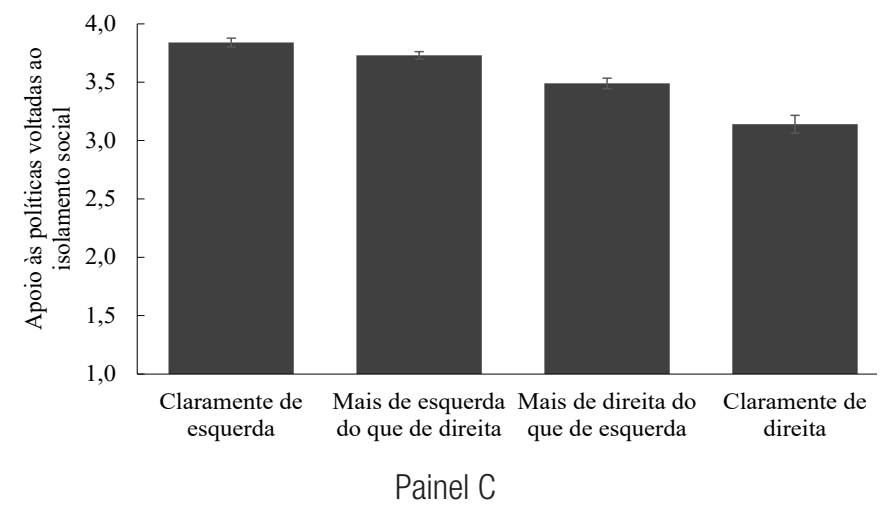

Probabilidade de doar para causas relacionadas à saúde

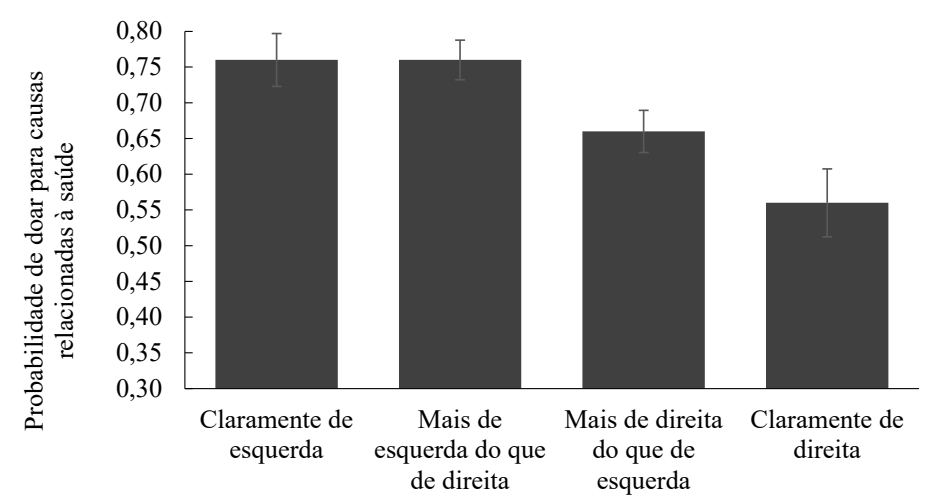

Fonte: Elaborada pelos autores.

Economia versus Saúde. Conforme destacado na introdução do artigo, pessoas que se reconhecem como de direita ou de esquerda demonstram diferenças fundamentais em valores e sistemas de crenças. É provável que, em comparação com o indivíduo de esquerda, o de direita dê mais peso aos efeitos econômicos negativos do isolamento social ao confrontar-se com o aparente trade-off entre as consequências da doença para a saúde e os efeitos econômicos da adoção do isolamento social. As diferenças significativas nas preferências sobre doar para uma ou outra causa (Figura 1 - Painel C) estão alinhadas com essa suposição. 
Nesse sentido, também examinamos se o menor apoio às práticas e políticas de isolamento social e as menores taxas de doações para causas relacionadas à saúde entre as pessoas que se consideram de direita poderiam ser explicadas por uma maior sensibilidade relativa às possíveis ameaças ao sistema econômico. Embora seja importante reconhecer que não tínhamos antecipado essa possibilidade no pré-registro do estudo, os dados coletados permitiram testá-la empiricamente.

Conforme descrito no item de procedimento, coletamos medidas que avaliam a sensibilidade relativa dos participantes às ameaças econômicas (sendo 1 para 'finanças', e 0 para 'saúde ou segurança') nos níveis pessoal, da comunidade próxima, ou da sociedade como um todo. Para analisar a importância relativa da sensibilidade às ameaças ao sistema econômico, realizamos uma série de regressões aparentemente não relacionadas com 5.000 replicações para testar modelos de mediação paralelos para cada uma das variáveis dependentes.

Como mostra a Figura 2 (Painéis A-C), e em consonância com a lógica que perpassa o estudo, a probabilidade de pessoas de direita (em comparação com as de esquerda) de optarem pela doação a causas relacionadas a economia foi significativamente mediada pela sensibilidade relativa às ameaças econômicas à sociedade $\left(\beta_{\text {sociedade }}=-0,03 ; z=-3,94 ;\right.$ bootstrap IC $\left.95 \%=[-0,043 ;-0,014]\right)$, mas não por ameaças econômicas a si próprio ou à comunidade $\left(\beta_{\text {pessoal }}=-0,01 ; z=-1,48 ;\right.$ bootstrap IC $95 \%=$ $[-0,016 ; 0,002] ; \beta_{\text {comunidade próxima }}=-0,01 ; z=-1,91 ;$ bootstrap IC $\left.95 \%=[-0,016 ; 0,000]\right)$. Além disso, a sensibilidade relativa dos participantes às ameaças econômicas à sociedade media significativamente a relação entre a orientação política e as práticas de isolamento social $\left(\beta_{\text {sociedade }}=-0,02 ; z=-3,04\right.$; bootstrap IC 95\% = [-. 041, -.009]), já as evidências dos efeitos no nível pessoal e da comunidade próxima foram mistas $\left(\beta_{\text {pessoal }}=-0,02 ; z=-2,18 ;\right.$ bootstrap IC $95 \%=[-0,031 ;-0,002] ; \beta_{\text {comunidade próxima }}$ $=-0,01 ; \mathrm{z}=-1,68 ;$ bootstrap IC $95 \%=[-0,019 ; 0,001])$. Finalmente, $\mathrm{o}$ efeito da orientação política no apoio às políticas de isolamento social foi mediado pela relativa sensibilidade dos participantes às ameaças econômicas à sociedade $\left(\beta_{\text {sociedade }}=-0,02 ; \mathrm{z}=-2,77\right.$; bootstrap IC 95\% $\left.=[-0,035 ;-0,006]\right)$, mas as evidências para os efeitos em níveis mais pessoais não alcançaram significância ( $\beta_{\text {pessoal }}=$ $-0,01 ; \mathrm{z}=-1,43 ;$ bootstrap $95 \%$ IC $=[-0,020,0,003] ; \beta_{\text {comunidade próxima }}=-0,005 ; \mathrm{z}=-1,07 ;$ bootstrap IC $95 \%=[-0,013 ; 0,004])$. Tomadas em conjunto, essas descobertas convergem com a ideia de que a orientação política molda o apoio ao isolamento social por meio de valores e sistemas de crenças mais gerais, em detrimento de motivações mais pessoais (isto é, preocupação com impactos econômicos do isolamento social para si próprio ou para uma comunidade próxima).

\section{FIGURA 2 (PAINÉIS A-C) - 0 PAPEL MEDIADOR DA SENSIBILIDADE RELATIVA ÀS AMEAÇAS ECONÔMICAS}

Painel A

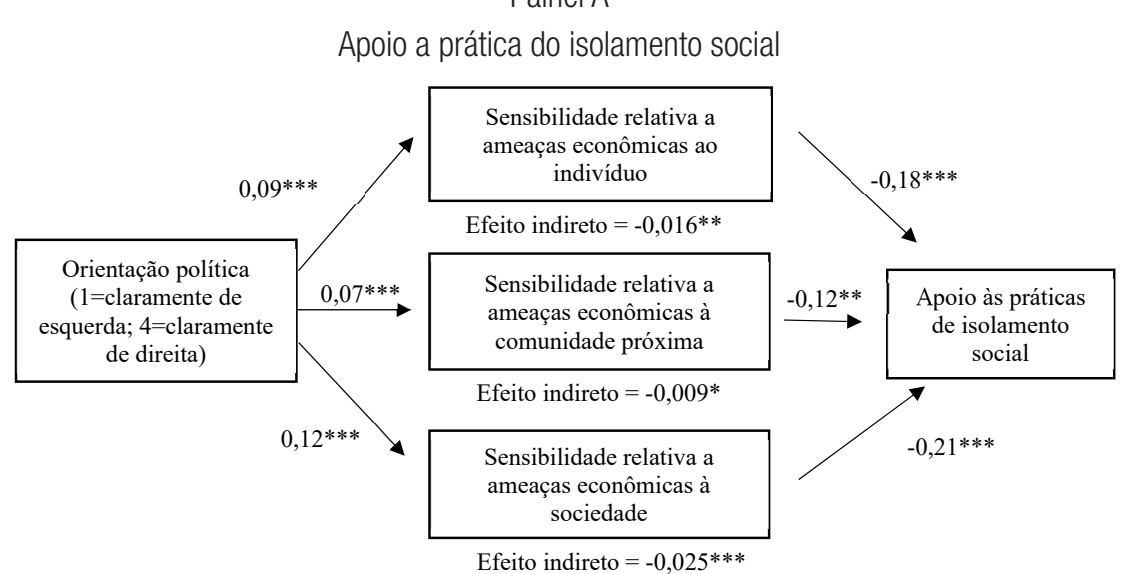


Painel B

Apoio às políticas de isolamento social

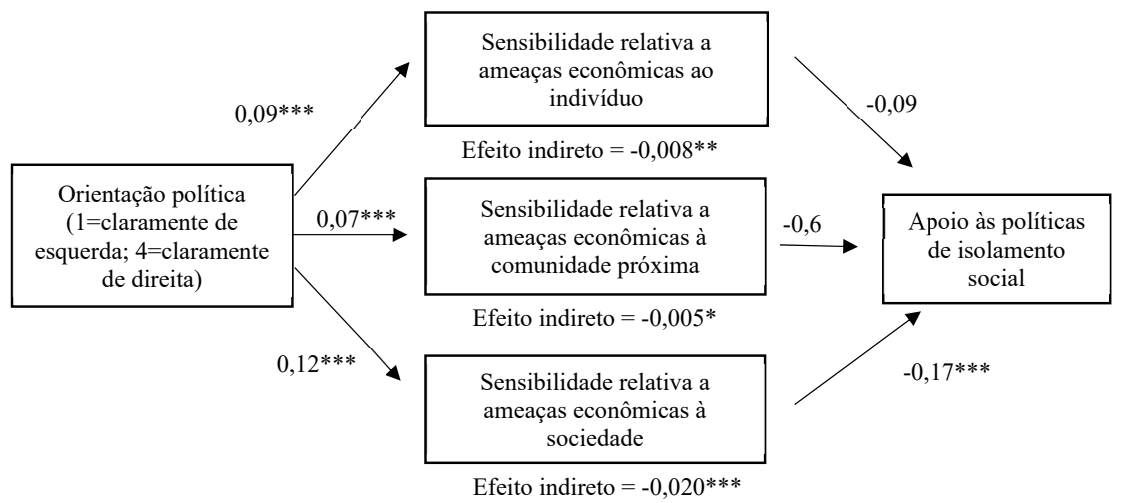

Painel C

Probabilidade de doar para causas relacionadas à saúde

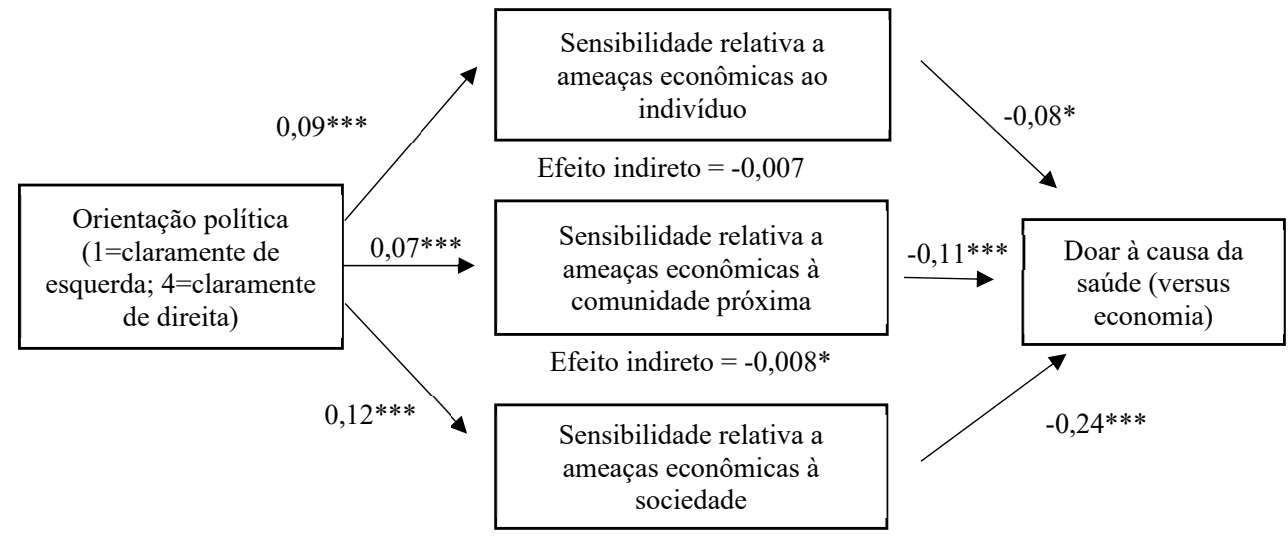

Efeito indireto $=-0,029 * * *$

Fonte: Elaborada pelos autores.

\section{DISCUSSÃO}

O surto da COVID-19 gerou um número impressionante de mortes em todo o mundo. No entanto, com base no argumento de que medidas rigorosas e antecipadas de isolamento social podem ser tão ou mais prejudiciais para a sociedade do que políticas de isolamento mais flexíveis - ou mesmo nenhuma política (Snooks, 2020), muitos cidadãos relutaram em aderir e/ou apoiar o isolamento social, apesar de um amplo consenso médico. Embora essa resistência possa estar fundamentada nos significativos custos econômicos pessoais advindos de tal política, é também possível que a falta de apoio ao isolamento social reflita princípios normativos e sistemas de crenças mais abstratos, cujo núcleo é a orientação política (Jost et al., 2009). Nosso estudo investigou a importância relativa da orientação política, em comparação com à vulnerabilidade econômica, em prever a atitude dos indivíduos em relação ao isolamento social e a decisão de doar para a causa da saúde (ou uma causa na área da economia). Corroborando nossas previsões, a orientação política se mostrou um significante preditor do apoio ao isolamento social, para além da vulnerabilidade econômica pessoal, que, surpreendentemente, não influenciou com robustez o apoio ao isolamento social. Mais especificamente, os indivíduos que se reconheceram como 'de direita' apoiaram sistematicamente menos as práticas e políticas de isolamento social quando comparados 
aos 'de esquerda'. Além disso, em um teste mais explícito do trade-off entre saúde pública e bem-estar econômico, a orientação política também se apresentou como preditor da decisão de doar para uma causa que visa mitigar os impactos da COVID-19 na saúde (versus na economia). As análises de mediação deram apoio adicional à noção de que o fenômeno está enraizado em sistemas de crenças abstratas, e não na vulnerabilidade econômica pessoal.

Reconhecemos que esse não é um estudo pioneiro em examinar a conexão entre orientação política e apoio ao isolamento social. Pesquisas emergentes no contexto da pandemia da COVID-19 dão suporte consistente a essa conexão e ao papel preditor da orientação política (Alcott et al., 2020; Barrios \& Hochberg, 2020; Conway III et al., 2020; Painter \& Qiu, 2020; Rosenfeld et al., 2020). De uma maneira mais geral, a pesquisa sobre orientação política e apoio a políticas públicas demonstra que as pessoas tendem a valorizar as iniciativas patrocinadas por seu próprio grupo político e a desvalorizar àquelas defendidas pelo grupo político oposto (Gadarian, Goodman, \& Pepinsky, 2020; Van Boven, Ehret, \& Sherman, 2018). Em contraste com essa linha de pesquisa, focamos no papel dos princípios normativos abstratos e dos sistemas de crenças que nutrem a orientação política. Ainda, pesquisas anteriores mostraram que a polarização política de uma determinada política pública ocorre ou não dependendo de características idiossincráticas regionais (McCright, Dunlap, \& Marquart-Pyatt, 2016). Enquanto a evidência existente do efeito da orientação política na adesão às medidas de isolamento social seja predominantemente baseada em dados dos EUA, movemos o debate para longe daquele contexto e investigamos a robustez do fenômeno no Brasil.

Nosso estudo também tem implicações práticas importantes para a comunicação pública. Os indivíduos que se consideram 'de direita' tendem a ser menos favoráveis ao isolamento social, porque são mais sensíveis às ameaças econômicas que essa medida aparentemente impõe à sociedade. Embora essa pandemia pareça ter consequências negativas inevitáveis para a economia, pesquisas anteriores mostraram que a adoção precoce e rigorosa de medidas como o isolamento social realmente produz efeitos positivos no crescimento econômico após a pandemia e, portanto, contribui para uma recuperação econômica mais rápida (Correia, Luck, \& Verner, 2020). Assim, em vez de aumentar a depressão econômica, a adoção de medidas de isolamento social parece atenuá-la, rompendo com o paradigma do trade-off. Os formuladores de políticas podem, portanto, destacar esse recurso nas comunicações públicas, a fim de aumentar a conformidade e o apoio ao isolamento social entre os indivíduos que se reconhecem como 'de direita'.

Como limitação da presente pesquisa, é importante ressaltar, em primeiro lugar, que a amostra não é representativa da população brasileira. Dada a urgência do problema e as restrições de coleta de dados impostas pelo vírus, a grande maioria dos estudos sobre a pandemia da COVID-19 compartilha essa limitação (Di Lorenzo et al., 2020; Marta et al., 2020; Padala, Jendro, \& Padala, 2020). Portanto, são necessárias coletas de dados em âmbito nacional com amostras representativas para atestar a validade externa do fenômeno aqui examinado. Segundo, o forte apoio de nossa amostra ao isolamento social sugere a existência de efeito teto. Assim, é recomendável que pesquisas futuras adotem medidas alternativas de apoio ao isolamento social para mitigar essa limitação. Terceiro, os dados coletados foram obtidos em um único período, em um momento extraordinário. Portanto, não apenas os resultados desta investigação justificam sua replicação, mas também as investigações futuras devem levar em conta possíveis variações, tanto nas direções que as políticas públicas possam tomar, como na percepção social da pandemia. No entanto, nossas descobertas mostram claramente o poder da orientação política - para muito além de outros aspectos como as considerações econômicas pessoais, por exemplo - de prever as atitudes e comportamentos relativos a proteção da saúde durante a pandemia da COVID-19 no Brasil. 


\section{REFERÊNCIAS}

Abramowitz, A. I., \& Saunders, K. L. (2008). Is polarization a myth?. The Journal of Politics, 70(2), 542-555.

Adler, N. E., Epel, E. S., Castellazzo, G., \& Ickovics, J. R. (2000). Relationship of subjective and objective social status with psychological and physiological functioning: Preliminary data in healthy, White women. Health psychology, 19(6), 586.

Allcott, H., Boxell, L., Conway, J., Gentzkow, M., Thaler, M., \& Yang, D. Y. (2020). Polarization and public health: Partisan differences in social distancing during the Coronavirus pandemic (NBER Working Paper). Cambridge, MA: The National Bureau of Economic Research.

Atieh, J. M., Brief, A. P., \& Vollrath, D. A. (1987). The Protestant work ethic-conservatism paradox: Beliefs and values in work and life. Personality and Individual Differences, 8(4), 577-580.

Bail, C. A., Argyle, L. P., Brown, T. W., Bumpus, J. P., Chen, H., Hunzaker, M. F., ... \& Volfovsky, A. (2018). Exposure to opposing views on social media can increase political polarization. Proceedings of the National Academy of Sciences, 115(37), 9216-9221.

Barrios, J. M., \& Hochberg, Y. (2020). Risk perception through the lens of politics in the time of the covid-19 pandemic (NBER Working Paper). Cambridge, MA: The National Bureau of Economic Research.

Carothers, T., \& O’Donohue, A. (Eds.). (2019). Democracies divided: The global challenge of political polarization. Washington, DC: Brookings Institution Press.

Choma, B. L., Hanoch, Y., Hodson, G., \& Gummerum, M. (2014). Risk propensity among liberals and conservatives: The effect of risk perception, expected benefits, and risk domain. Social Psychological and Personality Science, 5(6), 713-721.

Converse, P. E. (1964). “The Nature of Belief Systems in Mass Publics.” In D. E. Apter (Ed.), Ideology and Its Discontents (pp. 206-261). New York, NY: The Free Press of Glencoe.

Conway III, L. G., Woodard, S. R., Zubrod, A., \& Chan, L. (2020, 13 de abril). Why are conservatives less concerned about the coronavirus (COVID-19) than liberals? Testing experiential versus political explanations. PsyArXiv.
Correia, S., Luck, S., \& Verner, E. (2020, 05 de junho). Public Health Interventions Do Not: Evidence from the 1918 Flu (Working Paper). SSRN.

Di Lorenzo, G., Toniolo, P., Lurani, C., Foresti, L., \& Carrisi, C. (2020). Evaluating the adequacy of Prima Covid-19 IgG/IgM Rapid Test for the assessment of exposure to SARS-CoV-2 virus. medRxiv.

Dimock M, Carroll D. (2014). "Political polarization in the American public: How increasing ideological uniformity and partisan antipathy affect politics, compromise, and everyday life". Washington, DC: Pew Research Center.

Fiorina, M. F. P., Abrams, S. J. \& Pope, J. C. (2006). Culture War? The Myth of a Polarized America (2nd ed.). New York, NY: Pearson Longman.

Gadarian, S. K., Goodman, S. W., \& Pepinsky, T. B. (2020, March 27). Partisanship, health behavior, and policy attitudes in the early stages of the COVID-19 pandemic (Working Paper). SSRN.

Gramlich, J. \& Schaeffer, K. (2019). "7 facts about guns in the U.S.". Washington, DC: Pew Research Center.

Hasson, Y., Tamir, M., Brahms, K. S., Cohrs, J. C., \& Halperin, E. (2018). Are liberals and conservatives equally motivated to feel empathy toward others?. Personality and Social Psychology Bulletin, 44(10), 1449-1459.

Jacoby, W. G. (2000). Issue framing and public opinion on government spending. American Journal of Political Science, 750-767.

Jost, J. T., Federico, C. M., \& Napier, J. L. (2009). Political ideology: Its structure, functions, and elective affinities. Annual review of psychology, 60, 307-337.

Kerlinger, F. (1984). Liberalism and conservatism: The nature and structure of social attitudes. Hillsdale, NJ: Lawrence Erlbaum.

Korndörfer, M., Egloff, B., \& Schmukle, S. C. (2015). A large scale test of the effect of social class on prosocial behavior. PloS one, 10(7), e0133193.

Lancet, T. (2020). COVID-19: learning from experience. Lancet (London, England), 395(10229), 1011.

Loewenstein, G., Read, D., \& Baumeister, R. F. (Eds.). (2003). Time and decision: Economic and 
psychological perspectives of intertemporal choice. New York, NY: Russell Sage Foundation.

Mansbridge. (2016, 11 de março). Three reasons political polarization is here to stay. The Washington Post. Recuperado de https://www.washingtonpost. com/news/in-theory/wp/2016/03/11/three-reasonspolitical-polarization-is-here-to-stay/

Marta, I., Mirjana, Đ., Aleksandar, K., Filip, M., Kristina, N., Tamara, P., ... Irena, T. (2020). Serbian Citizens' Opinion on the COVID-19 Epidemic. South Eastern European Journal of Public Health (SEEJPH). Recuperado de https://doi.org/10.4119/seejph-3459

McCright, A. M., Dunlap, R. E., \& Marquart-Pyatt, S. T. (2016). Political ideology and views about climate change in the European Union. Environmental Politics, 25(2), 338-358.

Miguel, E., Camerer, C., Casey, K., Cohen, J., Esterling, K. M., Gerber, A., ... Van der Laan, M. (2014). Promoting transparency in social science research. Science, 343, 30-31.

Munafò, M. R., Nosek, B. A., Bishop, D. V. M., Button, K. S., Chambers, C. D., du Sert, N. P., ... Ioannidis, J. P. A. (2017). A manifesto for reproducible science. Nature Human Behavior 1, 1-9.

Nosek, B. A., Ebersole, C. R., DeHaven, A. C., and Mellor, D. T. (2018). The preregistration revolution. Proceedings of the National Academy of Sciences, 18, 201708274.

O’Donoghue, T., \& Rabin, M. (2015). Present bias: Lessons learned and to be learned. American Economic Review, 105(5), 273-79.

Padala, P. R., Jendro, A. M., \& Padala, K. P. (2020). Conducting clinical research during the COVID-19 Pandemic: Investigator and participant perspectives. JMIR Public Health and Surveillance, 6(2), e18887.

Painter, M., \& Qiu, T. (2020, April). Political beliefs affect compliance with covid-19 social distancing orders (Working Paper) SSRN.
Rosenfeld, D. L., Rothgerber, H., \& Wilson, T. (2020). Politicizing the covid-19 pandemic: Ideological differences in adherence to social distancing. PsyArXiv.

Rudolph, T. J., \& Evans, J. (2005). Political trust, ideology, and public support for government spending. American Journal of Political Science, 49(3), 660-671.

Saad, L. (2012). "Conservatives Remain the Largest Ideological Group in U.S.” Recuperado de http:// www.gallup.com/poll/152021/conservativesremain-largest-ideological-group.aspx

Samuels, D., \& Zucco, C., Jr. (2014). The power of partisanship in Brazil: Evidence from survey experiments. American Journal of Political Science, 58(1), 212-225.

Snooks, G. D. (2020). Fight the Virus (COVID-19), Not the Economy!. Institute of Global Dynamic Systems (Working Paper, 20). Canberra, Australia: Institute of Global Dynamic Systems.

Tabari, P., Amini, M., Moghadami, M., Moosavi, M. (2020). International Public Health Responses to COVID-19 Outbreak: A Rapid Review. Iranian Journal of Medical Sciences, 45(3), 157-169.

Tanne, Janice Hopkins (2020), “Covid-19: cases grow in US as Trump pushes promise of a malaria drug." BMJ (Clinical research ed.), 368, m1155.

Van Boven, L., Ehret, P. J., \& Sherman, D. K. (2018). Psychological barriers to bipartisan public support for climate policy. Perspectives on Psychological Science, 13(4), 492-507.

World Health Organization (2020, janeiro), "Household transmission investigation protocol for 2019-novel coronavirus ( 2019-nCoV ) infection," EarlyInvestigations-2019, 1-31.

Yamada, Y. (2018). How to crack pre-registration: Toward transparent and open science. Frontiers in psychology, 9, 1831. 


\section{Guilherme Ramos}

https://orcid.org/0000-0003-3519-3861

Mestre em Administração; Doutorando em Administração na Escola Brasileira de Administração Pública e de Empresas da Fundação Getulio Vargas (FGV EBAPE). E-mail: guilhermedearamos@yahoo.com.br

\section{Yan Vieites}

https://orcid.org/0000-0001-5708-109X

Mestre em Administração; Doutorando em Administração da Escola Brasileira de Administração Pública e de Empresas da Fundação Getulio Vargas (FGV EBAPE). E-mail: yan.vieites@gmail.com

\section{Jorge Jacob}

https://orcid.org/0000-0001-6786-3706

Doutor em Administração; Pesquisador de Pós-Doutorado na Columbia University e na University of Virginia. E-mail: jr3815@columbia.edu

\section{Eduardo B. Andrade}

https://orcid.org/0000-0002-2930-8151

Doutor em Marketing; Professor de Psicologia do Consumidor na Escola Brasileira de Administração Pública e de Empresas da Fundação Getulio Vargas (FGV EBAPE). E-mail: eduardo.b.andrade@fgv.br 


\section{APÊNDICE}

\section{A HIPÓTESE DO VIÉS DO PRESENTE}

Parte da explicação para o fato de as pessoas potencialmente resistirem à adoção do isolamento social pode ser o "viés do presente" (O’Donoghue \& Rabin 2015). A implementação e a adesão ao isolamento social podem ser pensadas como um problema clássico de escolha intertemporal (Loewenstein, Read \& Baumeister, 2003) onde, enquanto o benefício da redução no número de mortes leva tempo para se concretizar, os governos e a sociedade devem arcar com o custo do isolamento social nos estágios iniciais do surto. Se as consequências da pandemia para a saúde ainda estão por vir, os cidadãos e os formuladores de políticas públicas podem sentir-se relutantes em incorrer nos custos psicológicos e financeiros do isolamento social - que são certos e antecipados - na esperança de um benefício futuro - que ainda é uma probabilidade. No presente trabalho, tentamos 'trazer o futuro para o presente', ou seja, induzimos um subconjunto de participantes a refletir sobre as consequências da pandemia para a saúde (prevendo o número de mortes ou simulando uma tomada de decisão médica moralmente complexa). Nossa hipótese previa que aumentar a saliência das consequências que a pandemia traz para a saúde acarretaria em aumento no apoio ao isolamento social.

Para testar a hipótese, participantes foram designados aleatoriamente para uma de quatro condições experimentais, em que eles (a) não foram expostos a nenhuma intervenção (condição de controle); (b) foram perguntados qual grupo de pacientes deveria ser priorizado caso os recursos nos hospitais fossem insuficientes (condição de decisão médica); (c) estimaram o número de vítimas da COVID-19 nos 5 dias subsequentes em vários países e, em seguida, foram apresentados com os dados reais (condição do número estimado de mortes - 5 dias); ou (d) fizeram exatamente a mesma estimativa para os 10 dias subsequentes (condição do número estimado de mortes - 10 dias). Todas essas intervenções foram desenhadas para aumentar a relevância das possíveis consequências da pandemia para a saúde das pessoas. Em seguida, os participantes passaram às variáveis dependentes e depois ao questionário sociodemográfico, seguindo a metodologia detalhada no corpo do artigo.

Resultados. Analisamos os efeitos das intervenções na decisão de doar usando regressão logística e nas outras duas medidas de isolamento social usando modelos de regressão linear (Tabela A1). Conforme ilustrado na Figura A1, nenhuma de nossas intervenções aumentou consistentemente o apoio ao isolamento social ou doações para a causa da saúde. Parte do motivo pode ser o fato de que, em nossa amostra, o apoio a práticas de isolamento social $\left(\mathrm{M}_{\text {controle }}=3,51 ; \mathrm{DP}=0,71\right)$ e políticas públicas $\left(\mathrm{M}_{\text {controle }}=3,58 ; \mathrm{DP}=0,66\right)$ foram bastante altos. A única exceção foi que os participantes na condição de 'decisão médica' tinham maior probabilidade de doar para uma causa relacionada à saúde do que aqueles na condição inicial $(\beta=0,63 ; z=2,91 ; p=0,004)$. Para essa métrica, os efeitos do teto foram menos prováveis (Condição de controle $=0,66$ ). Portanto, a hipótese foi refutada. 


\section{FIGURA A1 EFEITOS DAS INTERVENÇÕES NO APOIO AO ISOLAMENTO SOCIAL E DOAÇÕES À CAUSA} RELACIONADA À SAÚDE

Painel A

Apoio às práticas de isolamento social

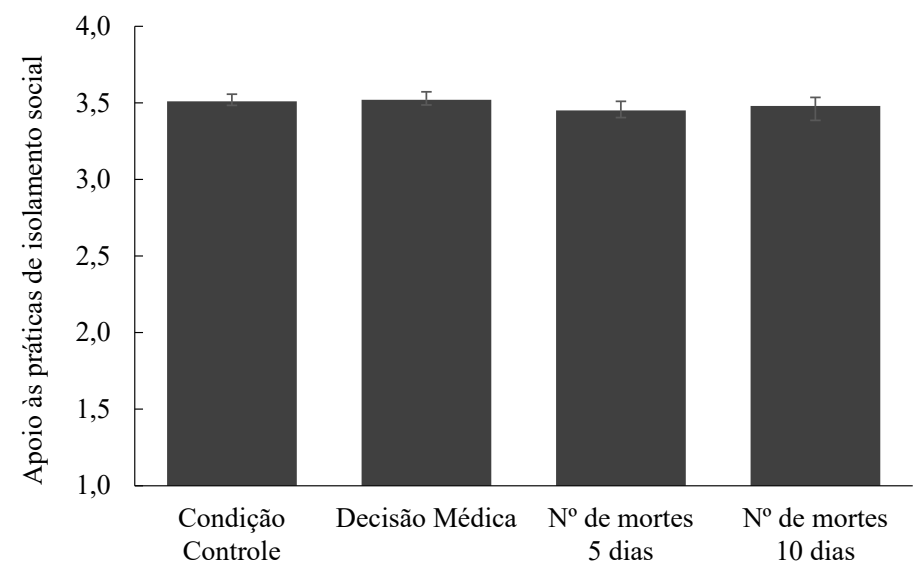

Painel B

Apoio às políticas públicas de isolamento social

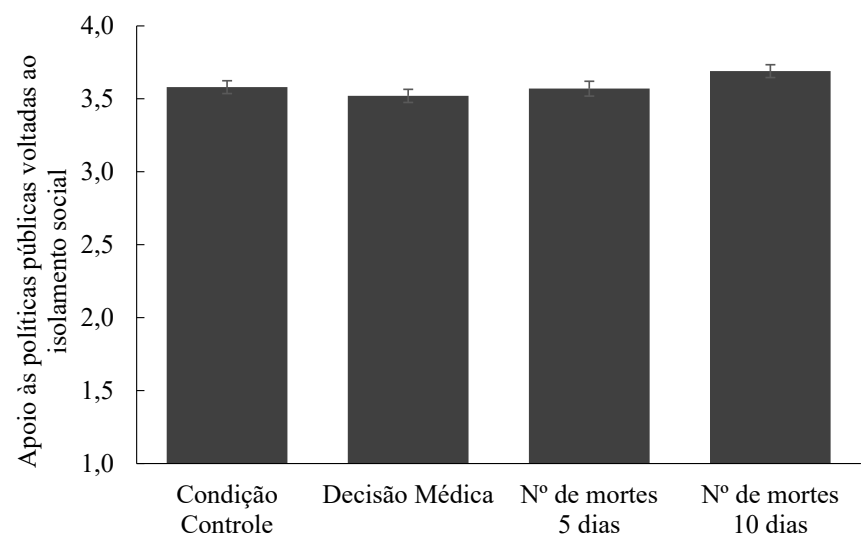

Painel C

Probabilidade de doar para causas relacionadas à saúde

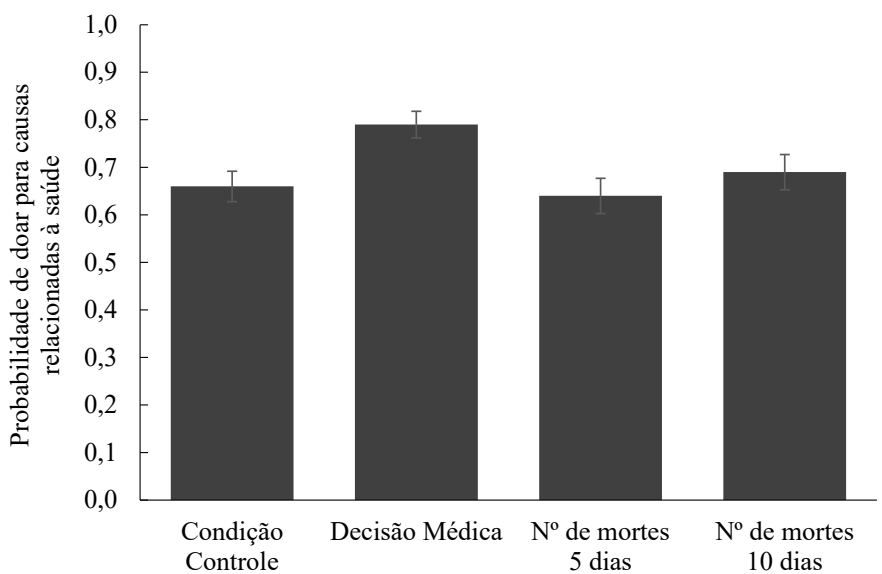

Fonte: Elaborada pelos autores. 
RAP | Orientação política e apoio ao isolamento social durante a pandemia da COVID-19: evidências do Brasil

\section{TABELA A1 EFEITO DAS INTERVENÇÕES NO APOIO AO ISOLAMENTO SOCIAL E NA ATITUDE DE DOAR PARA CAUSAS RELACIONADAS À SAÚDE}

\begin{tabular}{|c|c|c|c|}
\hline & \multicolumn{3}{|c|}{ Variável Dependente } \\
\hline & $\begin{array}{l}\text { Doar para causas } \\
\text { relacionadas à } \\
\text { saúde }\end{array}$ & $\begin{array}{l}\text { Apoio às práticas de } \\
\text { isolamento social }\end{array}$ & $\begin{array}{l}\text { Apoio às políticas de } \\
\text { isolamento social }\end{array}$ \\
\hline & Reg. Logística & MMQ & MMQ \\
\hline & (1) & (2) & (3) \\
\hline \multirow[t]{2}{*}{ Condição de decisão médica } & $0,63^{\star \star \star}$ & 0,01 & $-0,06$ \\
\hline & $(0,22)$ & $(0,07)$ & $(0,06)$ \\
\hline \multirow[t]{2}{*}{$\begin{array}{l}\text { Condição do } n^{0} \text { estimado de } \\
\text { mortes - } 10 \text { dias }\end{array}$} & 0,13 & $-0,02$ & $0,11^{*}$ \\
\hline & $(0,22)$ & $(0,08)$ & $(0,07)$ \\
\hline \multirow[t]{2}{*}{$\begin{array}{l}\text { Condição do } n^{0} \text { estimado de } \\
\text { mortes }-5 \text { dias }\end{array}$} & $-0,08$ & $-0,06$ & $-0,01$ \\
\hline & $(0,21)$ & $(0,08)$ & $(0,07)$ \\
\hline № Observações & 768 & 768 & 768 \\
\hline R-quadrado & & 0,00 & 0,01 \\
\hline Log-Probabilidade & $-464,34$ & & \\
\hline Crit. de inf. de Akaike & 936,69 & & \\
\hline
\end{tabular}

Nota: Erro padrão entre parênteses. ${ }^{* *} \mathrm{p}<0,1 ;{ }^{* *} \mathrm{p}<0,05 ;{ }^{\star} \mathrm{p}<0,10$.

Fonte: Elaborada pelos autores. 